\title{
Análisis de la dinámica económica, laboral y social en el municipio de Ocaña
}

\section{Analysis of the economic, labour and social dynamics in the municipality of Ocaña}

\author{
Cindy Paola Pineda - López ${ }^{1}$, Genny Torcoroma Navarro - Claro ${ }^{2}$ \\ ${ }^{1}$ Grupo GIDCOS, Corporación de Gestión Empresarial, Investigativa y Proyectos, Colombia, \\ ORCID: https://orcid.org/0000-0002-6149-7925, E-mail: cipapilo.04@gmail.com \\ ${ }^{2}$ Universidad Francisco de Paula Santander Ocaña, Colombia, \\ ORCID: https://orcid.org/0000-0003-3225-9028, E-mail: gtnavarroc@ufpso.edu.co
}

Cómo citar: Pineda- López, C. P., \& Navarro- Claro, G. T. (2019). Análisis de la dinámica económica, laboral y social en el municipio de Ocaña. Revista Científica Profundidad Construyendo Futuro, 10(10), 24-30. https://doi.org/10.22463/24221783.2518

Recibido: 28 de septiembre de 2018/ Aprobado: 15 de octubre de 2018

\begin{abstract}
Resumen
La dinámica económica, laboral y social, es un aspecto que genera grandes controversias, con el paso de los años se han producido cambios profundos que afectan la economía y más aún cuando su capacidad para generar crecimiento y empleo limita la creación de entornos prósperos que promuevan el establecimiento de empresas y políticas públicas que apoyen a las comunidades a mantenerse en equilibro. Por tanto, el propósito del estudio se centra en conocer la dinámica económica, laboral y social del municipio de Ocaña Norte de Santander, con relación a los gastos que se originan en las familias ocañeras, las condiciones de trabajo, así como, los problemas más recurrentes en la comunidad. La metodología utilizada fue de tipo cuantitativo, de alcance descriptivo, empleando como técnica la encuesta, aplicada a 300 personas. Los resultados parciales indican que en el municipio de Ocaña las familias cuentan con una baja capacidad para cubrir gastos y cada vez son más los problemas de orden público que se presentan en los diferentes barrios de la ciudad, impidiendo que se logren niveles de crecimiento sostenido que generen empleo, ingresos y una mejor calidad de vida.
\end{abstract}

Palabras clave: Condiciones laborales, Calidad de vida, Dinámica económica, Problemas de orden público.

\begin{abstract}
The economic, labor and social dynamics, is an aspect that generates great controversy, over the years there have been profound changes that affect the economy and even more so when its ability to generate growth and employment limits the creation of prosperous environments that promote the establishment of businesses and public policies that support communities to stay in balance. Therefore, the purpose of the study is to understand the economic, labor and social dynamics of the municipality of Ocaña Norte de Santander, in relation to the expenses that originate in the Ocaña families, the working conditions, as well as, the most recurrent problems in the community. The methodology used was quantitative, with a descriptive scope, using the survey technique applied to 300 people. The partial results indicate that families in the municipality of Ocaña have a low capacity to cover expenses and that there are more and more problems of public order in the different neighbourhoods of the city, preventing the achievement of sustained growth levels that generate employment, income and a better quality of life.
\end{abstract}

Keywords: Working conditions, Quality of life, Economic dynamics, Problems of public order. 


\section{Introducción}

La dinámica económica en las ciudades y regiones es un proceso que se revisa a partir del crecimiento sostenido, es decir, con la generación de empleo, para que las familias puedan obtener ingresos que les ayuden a solventar sus necesidades y por ende mantener una buena calidad de vida; incluso, autores como Raúl Prebisch (1901-1986), citado en (Pérez y Vernengo, 2016), sostiene que para que se dé un adecuado proceso productivo, es necesario realizar ciertos cambios en la forma como se desarrollan los mismos, con el fin de que las actividades económicas, incluidos la producción, el empleo y la distribución, se mantengan en circulación dado que todo se mantiene bajo un mismo ciclo.

En esta medida, la generación de políticas públicas capaces de contrarrestar las problemáticas en las que se halla sumergida la sociedad es clave para la construcción de territorios prósperos, capaces de crear escenarios donde prime no solo el bienestar de algunos, sino también, de todo un conglomerado, y así, minimizar el desequilibrio presente entre las masas, el cual incide en gran medida dentro de la economía y el mercado laboral.

De manera que, el comenzar a crear nuevas y mejores oportunidades para que las personas desarrollen sus capacidades para innovar y generar riqueza, hace que se creen entornos más sanos y prósperos; por consiguiente, el dinamizar la economía conlleva al fortalecimiento de toda una sociedad, tomando como punto de partida factores psicológicos y naturales, pues son los que permiten que una comunidad se acople a los ciclos económicos.
Mientras tanto, si los gobiernos locales no desarrollan una configuración oportuna y cíclica a todas aquellas variables que se encuentra dispersas en las comunidades como lo son la calidad de vida, el número de miembros en los hogares, las condiciones laborales, las problemáticas existentes en cada localidad, etc. las cuales inciden directa e indirectamente en su desarrollo, llegando en ocasiones a afectarles de manera positiva o negativa, es lo que ha hecho que al momento de diseñar planes, programas y proyectos que coadyuven en su bienestar no son del todo efectivos (López, Ávila y Mendez, 2011).

Partiendo de lo anterior, por medio de la investigación se logra analizar la dinámica económica, laboral y social en el municipio de Ocaña, así como, las principales problemáticas que aquejan y limitan el correcto crecimiento y desarrollo de la sociedad.

\section{Marco teórico}

El presente apartado posee teorías que respaldan la construcción de los resultados.

\subsection{Teoría de la dinámica económica}

La dinámica económica en la sociedad es una cuestión que varía constantemente, pues el ciclo de producción en el que se encuentran sumergidos cada uno de los sectores de la economía, opera bajo variables tanto dependientes como independientes, de allí, que el establecer planes y programas que ayuden a generar un comportamiento económico coherente con la concepción humana, permite tener un equilibrio entre ambos; por tal razón, se profundiza sobre esta teoría la incidencia que tiene el realizar expansiones y contracciones para la producción. Ahora, para que se dé un 
crecimiento optimo y oportuno, es fundamental que se generen aumentos progresivos y constantes de los recursos monetarios, para completar dicho ciclo, pero para ello es conveniente que tanto los gastos, oferta y consumo se den por igual, ya que una inclinación hacia uno solo de estos elementos de que se compone la economía conlleva a una alteración en el nivel de crédito, el cual, no es el mismo en una economía dinámica que en un estacionaria (Deneeve, 2009).

\subsection{Teoría de los ciclos económicos}

Esta teoría, parte del hecho de que para lograr que la actividad productiva se acople a los ciclos económicos, deben tomarse en cuenta factores que van desde comportamiento psicológico, hasta fenómenos de corte natural, donde el aumentar la cantidad de dinero circulante y su valor es la única forma de sobrellevar los niveles de producción.

Por consiguiente, es importante que las organizaciones tomen en consideración cada uno de los determinantes exógenos y endógenos del ciclo económico, a fin de lograr plantear acciones estratégicas que conduzcan a la obtención de crecimiento y desarrollo, por ende, la inclusión de tecnologías dentro de los sistemas de producción se ha convertido en una pieza clave en la actividad productiva (Franco, 2001).

\section{Metodología}

La metodología empleada fue descriptiva con un enfoque cuantitativo, dado que al obtener los resultados se realizaron análisis que permitieron conseguir datos para resolver los interrogantes sobre la dinámica económica, laboral y social en el municipio de Ocaña Norte de Santander. La investigación es probabilística puesto que todos los elementos de la población tienen una misma probabilidad de ser seleccionados en la muestra (Hernández R. , 2014). Finalmente, es de anotar que como instrumento se empleó la encuesta y como instrumento el cuestionario, en la que participaron 300 personas de los diferentes barrios de la ciudad de Ocaña.

\section{Resultados}

En este apartado se exponen como se encuentra la dinámica económica, laboral y social en el municipio de Ocaña Norte de Santander, y se exhiben algunas de las razones por las que la comunidad ocañera alude en la ciudad no se ha logrado tener un buen crecimiento y desarrollo.

Más de $11 \quad 0 \%$

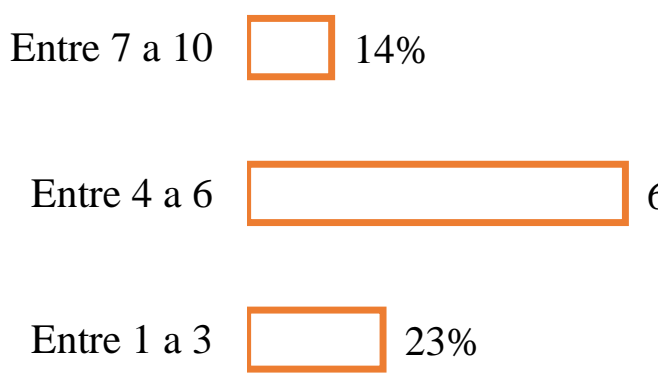
$63 \%$

Figura 1. Número de miembros en el hogar. Fuente: autores de la investigación.

Como se puede apreciar, en la ciudad de Ocaña el número de miembros en los hogares es significativo, puesto que en la mayoría hay entre 4 a 6 personas (63\%), sin embargo, existen viviendas en donde el número de miembros no supera las tres personas $(23 \%)$, aunque en algunos casos es más alto, pues en 
un mismo hogar puede haber entre 7 a 10 personas $(14 \%)$.

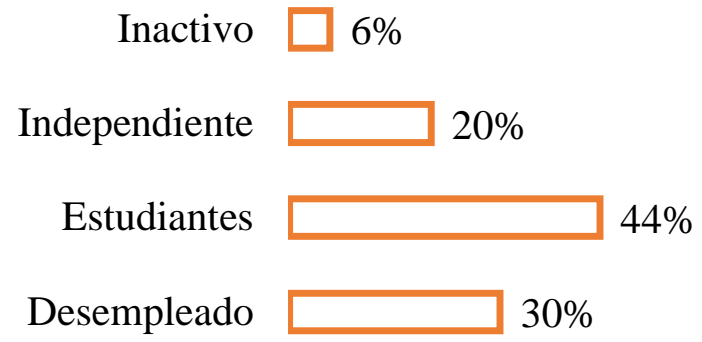

Figura 2. Condiciones laborales. Fuente: autores de la investigación.

La situación laboral es un aspecto que aqueja hoy día a muchas personas en el país, pues no cuentan con un empleo estable para poder suplir sus necesidades, y más cuando en el hogar hay personas que dependen del salario para poder sobrevivir.

Partiendo de lo anterior, y como se observa en la figura 2, en los hogares Ocañeros un $44 \%$ de sus miembros son estudiantes, un $30 \%$ no posee un empleo, mientras que un $20 \%$ se dedica a trabajar como independiente; finalmente un $6 \%$ de estos se encuentra inactivo, es decir, que estando en edad para trabajar no lo realiza.

$\square \mathrm{NO} \square \mathrm{SI}$

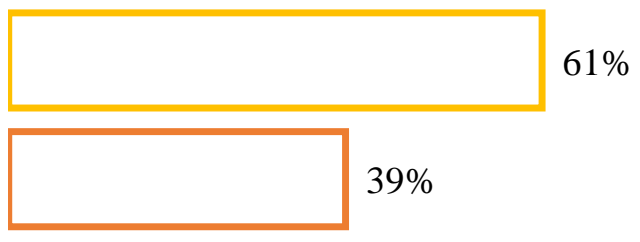

Figura 3. Capacidad para cubrir gastos. Fuente: autores de la investigación.
En relación a los datos arrojados por el instrumento, se obtuvo que en la ciudad de Ocaña las familias no cuentan con una buena capacidad económica para cubrir sus gastos, pues tan solo un 39\% de los encuestados manifiesta que los ingresos que poseen son suficientes para solventar en su totalidad los gastos que se producen constantemente en el hogar. Incluso, el hecho de que de un mes a otro los gastos aumenten como se muestra en la figura 4, produce en las familias ocañeras limitaciones frente a otras necesidades que aun cuando no son de primera necesidad son necesarias para mantener su equilibro emocional.

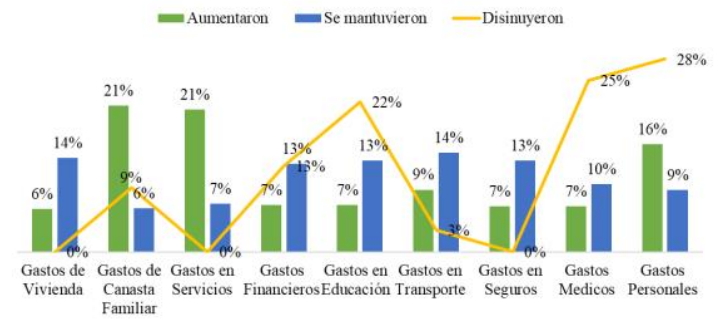

Figura 4. Gastos con relación al mes anterior. Fuente: autores de la investigación.

En efecto, el no contar con los recursos suficientes para sufragar los gastos se debe en gran medida a que la dinámica económica en la ciudad es volátil, pues como se puede apreciar en la figura 5, existen factores como el microcomercio, el desempleo, los impuestos, la competencia desleal, los pecios de la canasta familiar, entre otros, que en ciertas oportunidades aumentan, en otras se mantienen, mientras que en otras disminuyen.

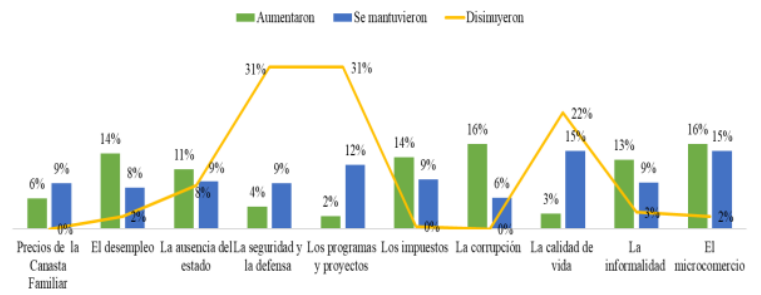

Figura 5. Dinámica económica. Fuente: autores de la investigación. 
Como se puede observar, los factores que han disminuido en mayor proporción con relación a la dinámica económica en el municipio de Ocaña, es la seguridad y defensa, los programas y proyectos, al igual que la calidad de vida.

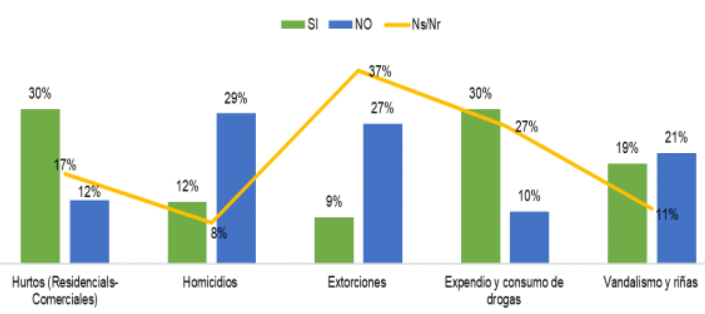

Figura 6. Problemas de orden público. Fuente: autores de la investigación.

Los problemas de orden público en el municipio de Ocaña, es uno de los aspectos más controversiales pese a los esfuerzos realizados por el gobierno local y las autoridades, en los diferentes barrios de la ciudad continúan presentándose en gran medida, pues como se puede apreciar en la figura $6, \quad$ los hurtos residenciales $y$ comerciales, al igual que el expendio y consumo de drogas, siguen en aumento.

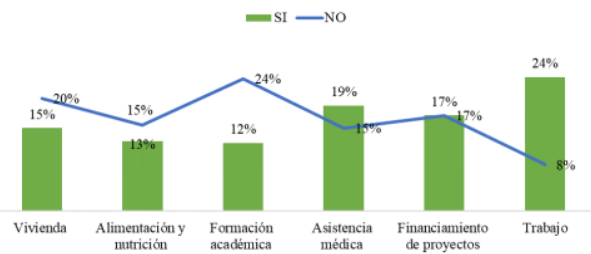

Figura 7. Problemáticas existentes en la comunidad. Fuente: autores de la investigación.

Tras analizar los datos obtenidos con el instrumento a la comunidad del municipio de Ocaña, se determina que las problemáticas que más afrontan las familias son en temas relacionados con el trabajo (24\%) y la asistencia médica (19\%), en primera instancia, porque son muy pocas las oportunidades de empleo que se brindan y en segunda medida porque no existe priorización en la asistencia de la comunidad.

\section{Conclusiones y discusiones}

La dinámica económica tanto a nivel local como nacional sigue siendo débil, y más cuando se generan cambios profundos en el ciclo económico que disminuye el desempeño de cada uno de los elementos que lo conforman, pues a partir del estudio, se logra determinar que, en el municipio de Ocaña, son muchos los aspectos que dificultan el progreso de las diferentes comunidades, como lo es la baja capacidad para cubrir gastos, el aumento y la disminución del desempleo, los impuestos, la competencia desleal, etc.

En coherencia con el planteamiento anterior, la dinámica económica, laboral y social, es una cuestión que debe ser tomada no como algo pasajero, sino, como un factor preponderante para lograr adquirir crecimiento, pues de acuerdo con lo establecido por la teoría monetaria y el ciclo comercial, cualquier cambio que se produzca por pequeño que sea genera que todos los bienes, ya sean de producción o consumo ocasionen incertidumbre y desequilibrio en la economía (Pérez y Vernengo, 2016); incluso existe aspectos que para muchos puede no ser tan importante para dinamizar una económica como el hábito de la lectura, pero que al estudiarlo a fondo puede traer a una comunidad no solo nuevas formas de establecer estrategias que ayuden a mejorar los procesos sino también su bienestar (Moreno, 2018).

De acuerdo con la Fundación Universitaria Jorge Tadeo Lozano (2015) es a raíz de los fuertes cambios que se han dado con el paso de los años que tanto la dinámica económica, 
laboral como social ha sido modificada sustancialmente, hasta el punto que las comunidades se han visto en la necesidad de establecer planes, programas y proyectos que les ayuden a sobrellevar las situaciones que se presentan a diario en cada una de sus localidades.

Por consiguiente, es fundamental que los gobiernos locales participen de manera activa en el desarrollo, inversión y crecimiento económico de las comunidades, para lograr mantener una estabilidad dentro de los niveles de ciclo económico, debido a que esto hace que la pobreza se reduzca (Laverde y Gómez, 2015).

Finalmente, Milanese (2004) indica que el rol que cumplen los gobiernos locales en las comunidades, es fundamental para aportar en la calidad de vida de cada una de las familias de las diferentes localidades, por lo que no solo basta con plantear acciones estrategias para mejorar el bienestar de la población, sino efectuando análisis mucho más profundos respecto a las variables endógenas y exógenas que se dan en la comunidad ya sean positivas o negativas, para de esta manera conocer a ciencia cierta el comportamiento de la dinámica económica, laboral y social en que se encuentra la ciudad, y así lograr establecer políticas públicas más reales y acorde a las necesidades.

\section{Referencias}

Deneeve, E. (2009). La teoría general de la dinámica económica de Bernard. Revista Universitas Philosophica, 26(53), 145-179. Obtenido de https://www.redalyc.org/pdf/4095/40 9534417008.pdf
Franco, H. (2001). La teoría del ciclo económico: El caso Colombiano las tres úlimas décadas. Revista Universidad EAFIT, 19-29. Obtenido de H Franco - Revista Universidad Eafit, 2001

publicaciones.eafit.edu.co

Fundación Universitaria Jorge Tadeo Lozano. (2015). Breve historia económica de Colombia (Primera edición ed.). (E. S. Kalmanovitz, Ed.) Bogotá: UTADEO. Obtenido de https://www.utadeo.edu.co/files/node /publication/field_attached_file/pdf_breve_historia_economica_de_colo mbia_ultimo_-_24-11-15.pdf

Hernández, R. (2014). Metodología de la investigación. McGRAW-HILL / INTERAMERICANA EDITORES, S.A. DE C.V.

Laverde, H., y Gómez, J. (2015). Medición de la pobreza multidimensional en América Latina a tráves de modelos estructurales. Obtenido de https://revistas.ucc.edu.co/index.php/ co/article/view/1130/1226

López, E., Ávila, A., y Mendez, G. (2011). Dinámica del mercado laboral colombiano: un análisis de políticas de empleo aplicando dinámica de sistemas. Obtenido de https://www.urosario.edu.co/Adminis tracion/documentos/9-

Dinamicas/017_1701714017/

Milanese, A. (2004). Dinámica de la ciudad. Revista Arte, Ciencia y Tecnología (PHAROS), 11(2), 61-90.

Moreno, E. C. (2018). Habitos que impiden la adaptación y adopción de la lectura en 


\section{Cindy Paola Pineda López, Genny Torcoroma Navarro Claro}

estudiantes de administración de empresas. Revista cientifica profundidad, 9(9). Obtenido de https://revistas.ufps.edu.co/index.php /profundidad/article/view/2457/2597

Pérez, E., y Vernengo, M. (2016). Raúl Prebisch y la dinámica económica: crecimiento cíclico e interacción entre el centro y la periferia. Revista Comisión Económica para América Latina (CEPAL). Obtenido de E Pérez Caldentey, M Vernengo - Revista Cepal, 2016 - repositorio.cepal.org 